\title{
Electronic wave functions and optical transitions in (In,Ga)As/GaP quantum dots
}

\author{
C. Robert, ${ }^{1,2}$ K. Pereira Da Silva, ${ }^{3,4}$ M. O. Nestoklon, ${ }^{5}$ M. I. Alonso, ${ }^{3}$ P. Turban, ${ }^{6}$ J.-M. Jancu, ${ }^{1}$ J. Even, ${ }^{1}$ H. Carrère, ${ }^{2}$ \\ A. Balocchi, ${ }^{2}$ P. M. Koenraad, ${ }^{7}$ X. Marie,${ }^{2}$ O. Durand, ${ }^{1}$ A. R. Goñi,${ }^{3,8}$ and C. Cornet ${ }^{1}$ \\ ${ }^{1}$ Université Européenne de Bretagne, INSA, FOTON UMR 6082, 35708 Rennes, France \\ ${ }^{2}$ Université de Toulouse, INSA-CNRS-Université Paul Sabatier (UPS), Laboratoire de physique et chimie de nano-objets (LPCNO), \\ 135 Avenue Rangueil, 31077 Toulouse, France \\ ${ }^{3}$ Institut de Ciència de Materials de Barcelona, Consejo Superior de Investigaciones Científicas (ICMAB-CSIC), \\ Campus Universitat Autònoma de Bellaterra (UAB), 08193 Bellaterra, Spain \\ ${ }^{4}$ Departamento de Física, Universidade Federal do Ceará, P.O. Box 6030, Fortaleza-CE, 60455-970, Brazil \\ ${ }^{5}$ Ioffe Institute, St. Petersburg 194021, Russia \\ ${ }^{6}$ Institut de physique de Rennes (IPR), UMR 6251, CNRS-Université de Rennes I, Campus de Beaulieu 35042 Rennes Cedex, France \\ ${ }^{7}$ Department of Applied Physics, Eindhoven University of Technology, P.O. Box 513, 5600 MB Eindhoven, The Netherlands \\ ${ }^{8}$ Institució Catalana de Recerca i Estudis Avançats (ICREA), Passeig Lluís Companys 23, 08010 Barcelona, Spain
}

(Received 9 March 2016; revised manuscript received 19 July 2016; published 30 August 2016)

\begin{abstract}
We study the complex electronic band structure of low In content InGaAs/GaP quantum dots. A supercell extended-basis tight-binding model is used to simulate the electronic and the optical properties of a pure $\mathrm{GaAs} / \mathrm{GaP}$ quantum dot modeled at the atomic level. Transitions between hole states confined into the dots and several $X_{Z}$-like electronic states confined by the strain field in the GaP barrier are found to play the main role on the optical properties. Especially, the calculated radiative lifetime for such indirect transitions is in good agreement with the photoluminescence decay time measured in time-resolved photoluminescence in the $\mu$ s range. Photoluminescence experiments under hydrostatic pressure are also presented. The redshift of the photoluminescence spectrum with pressure is also in good agreement with the nature of the electronic confined states simulated with the tight-binding model.
\end{abstract}

DOI: 10.1103/PhysRevB.94.075445

\section{INTRODUCTION}

The integration of laser devices on silicon has attracted much interest in the past decade as it would open the way of truly integrated photonic layers on silicon [1]. Especially, the hybrid integration of III-V structures by bonding [2] or epitaxial lift-off and transfer-printing [3] are considered as very potent short-term solutions to address the medium volume applications of high-bandwidth data transmissions in servers and data centers. On a longer term, the monolithic integration of III-V semiconductors lasers on silicon is expected to match more surely with the very large scale integration requirements [4-6]. Unfortunately, crystalline defects generated during the III-V/Si heteroepitaxy are known to limit the laser device performances; a thick buffer layer is usually needed to avoid the emergence of these structural defects, which limits the optical coupling solutions with the silicon chip. Recently, it was proposed by several groups to use a pseudomorphic $\mathrm{GaP} / \mathrm{Si}$ template, benefiting from the low lattice mismatch between $\mathrm{GaP}$ and $\mathrm{Si}(0.37 \%$ at room temperature) to eliminate the formation of these structural defects [7-13]. In this approach, the structural benefit is, however, counterbalanced by the limited optical properties of GaP-based materials because of the GaP indirect bandgap. To solve this issue, GaAsPN quantum well nanostructures have already been widely studied by different groups, both theoretically and experimentally, from their fundamental properties to laser operation on silicon [14-19]. The quantum efficiency of these nanostructures is, however, intrinsically limited by the indirect bandgap of GaAs when strained to $\mathrm{GaP}$ or $\mathrm{Si}$, and the addition of nitrogen only leads to a fractional $\Gamma$ character of the optical transitions (i.e., a pseudodirect bandgap optical transition) [15].

The hope for reaching a true direct bandgap emission with nanostructures pseudomorphically integrated on silicon was recently given by the demonstration of room temperature photoluminescence (PL) of high density (In,Ga)As/GaP quantum dots (QDs) [20]. From these promising results, different devices based on (In,Ga)As/GaP QDs were considered, such as resonant photonic crystal cavities [21], storage devices [22], or electroluminescent devices on GaP or Si substrates [23,24]. (In,Ga)As/GaP QDs lasing was even recently reported on $\mathrm{GaP}$ substrate by Heidemann et al. [25]. Meanwhile, structural properties of these QDs were investigated at a large scale in terms of size and density [20,26-28] and at the atomic scale through transmission electron microscopy (TEM), planview, and cross-sectional scanning tunneling microscopy (STM) [29-32], revealing a complex and inhomogeneous indium incorporation behavior inside the QDs. Fukami et al. first noticed in their pioneering works the vicinity of the $\Gamma$ conduction band minimum in the QD and the $X$ valley of $\mathrm{GaP}$ with a simple model-solid theory approach [33]. The description of the electronic structure of (In,Ga)As/GaP QDs was then refined using a mixed eight-band $\mathrm{k} \cdot \mathrm{p} /$ tight-binding (TB) $s p d s^{*}$ methodology [27,29]. In these works, realistic quantum confinement effects were taken only into account at the $\Gamma$ point with the $\mathrm{k} \cdot \mathrm{p}$ method, while lateral valleys $(X$ and $L$ ) were described using a TB $s p d s^{*}$ extended orbital basis for a quantum well with equivalent thickness. Despite the imperfection of the theoretical methodology, this paper led to the important conclusion that a competition between $X$-like states and $\Gamma$ states does exist in the conduction band of 
(In, Ga)As/GaP and that tuning the In content or the QD size can help in promoting the direct optical transition, necessary for high performance devices on silicon. These theoretical predictions were also consistent with the experimental observation of two main optical transitions already described by continuous wave (cw) PL experiments in the early work of Nguyen Thanh et al. [20] and Robert et al. [28] and more deeply investigated in Refs. [27] and [29] by means of time-resolved PL (tr-PL). The low energy (LE) transition was at this time assigned to an $X$-like transition and the higher energy transition to a $\Gamma$ one. A significant improvement of the PL intensity was recently observed by playing with the QDs morphology and strain relief during the growth. It was attributed to the promotion of the direct bandgap transition, in good agreement with the previous theoretical description of the QDs' electronic structure [34]. Still, the previous electronic structure modeling is not satisfactory in such a complex system, especially in the low In content range. In order to take into account the simultaneous quantum confinement effects on $X, L$, and $\Gamma$ valleys and their possible mixing [29], in a situation where QD and barrier states are close to each other, a full supercell TB model was developed at the atomic level in a recent work on a pure GaAs/GaP QD [35]. While providing an accurate description of the eigenstates of the QD (with a detailed analysis of the contributions of $X_{Z}, X_{X Y}, \Gamma$, and $L$ valleys), this paper restricted itself to the description of the electrons and holes ground states. Moreover, optical properties were not derived from the calculations, giving no indication on the oscillator strength of the described electron-hole recombination.

In this paper, we present an analysis of both electrons and holes ground and excited eigenstates and associated optical transitions in a pure $\mathrm{GaAs} / \mathrm{GaP} \mathrm{QD}$, using full supercell $\mathrm{TB}$ calculations at the atomic level. The exact geometry of QD is first extracted from atomically resolved plan-view and crosssectional STM images. Electrons and holes wave functions are then computed and analyzed in terms of orbital and Bloch functions decomposition. Optical absorption spectrum is theoretically derived for this QD. Results are used to interpret experimental data of tr-PL at ambient pressure conditions and cw PL under hydrostatic pressure both performed on low In content (below 15\%) QDs. Finally, a scheme of the complex electronic band structure of these QDs is proposed.

\section{QD GEOMETRY AND COMPOSITION}

The precise knowledge of the QD shape was most of the time considered as a limiting factor for QD band structure calculations, thus raising unsolvable questions about the ability to fit experimental data with theoretical calculations. Moreover, only a limited choice of QD geometry could be described by using $\mathrm{k} \cdot \mathrm{p}$ calculations [27,36]. One of the advantages of the fully atomistic supercell TB model is that it does not require any approximation on the QD shape, leading to simulations using very realistic geometries. Moreover, recent progresses in STM or TEM high resolution imaging have provided a very precise atomistic description of the QD morphology, even when it is buried [29,30]. Figures 1(a) and 1(b) present the STM plan-view and cross-sectional view of low In content (In,Ga)As/GaP QDs. Cross-sectional STM
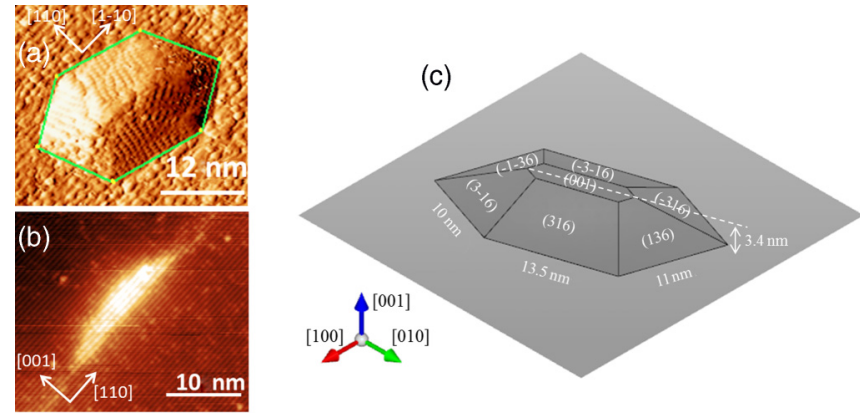

FIG. 1. Plan-view (a) and cross-sectional (b) STM images of (In,Ga)As/GaP QDs with a low In content. (c) QD geometry used for the tight-binding calculation.

(X-STM) measurements are performed on the (110) natural cleavage plane. These QDs have been grown by solid-source molecular beam epitaxy under the same conditions (3ML deposited at $580^{\circ} \mathrm{C}$ ), already reported in details in previous studies [20,29], except that a GaP capping layer is used for cross-sectional STM (X-STM), while QDs are uncapped for the plan-view imaging. From the plan-view image of Fig. 1(a), the morphology of the unburied QD can easily be defined at the atomic scale, analyzing angles of reconstructed facets with the nominal (001) plan, and QD edge direction angles with the [1-10] and [110] directions that is fixed by the dimer orientation on the (001) nominal surface [the wetting layer (WL), here]. Resulting geometry and crystallographic orientations are illustrated in Fig. 1(c). During the GaP capping process, the morphology of QDs may change due to the (In,Ga)As/GaP interplay. In this regard, a typical X-STM profile image taken on the (110) natural cleavage plane is shown in Fig. 1(b). In this image, the lateral dimension of the QD and the angles of the facets are similar to what was observed in plan-view imaging. We therefore assume in the following that the facets previously defined and the in-plane footprint of the QD remain unchanged during the capping process. On the other hand, the capped QD observed in cross-sectional STM has a slightly smaller height, with a smoother edge at the apex, as compared to the uncapped QD, due to mass transport during the capping process [37]. Therefore, the QD is truncated at $3.4 \mathrm{~nm}$ in the calculations, as illustrated in Fig. 1(c). A thin WL is also observed in Fig. 1(b); therefore, a 1-monolayer-(ML)-thick WL is added in the definition of the QD geometry for calculations.

The geometry of the QDs being almost known at the atomic scale, the question of its composition remains. Even if a two-dimensional (2D) mapping can be extracted from X-STM, giving the local composition of indium in the truncation plan of the QD [30], a full three-dimensional (3D) reconstruction of the $\mathrm{In} / \mathrm{Ga}$ ratio remains very tricky at the atomic scale [38-40]. Nevertheless, the average In content of the grown QDs is below $15 \%$, as already widely discussed in previous works [20,29]. We will therefore consider in the following pure GaAs/GaP QDs as a reference system for further theoretical studies dealing with the effects of inhomogeneous In incorporation. 


\section{ENERGY LEVELS, WAVE FUNCTIONS, AND OPTICAL ABSORPTION}

\section{A. Supercell construction}

An initial supercell is first built with the Ga, As, and $\mathrm{P}$ atoms, regularly placed on the sites of an unstrained zincblende structure with the GaP lattice constant and following the facets and edge directions presented in Fig. 1(c). Periodic conditions are applied at the borders of the supercell for both strain and band structure calculations. The lateral size of the supercell is around $36 \times 36 \mathrm{~nm}^{2}$, equivalent to a QD areal density of $7.7 .10^{10} \mathrm{~cm}^{-2}$. The thickness of the barrier material in the [001] direction is $86 \mathrm{GaP}-\mathrm{MLs}$, accounting for a $\mathrm{GaP}$ buffer layer below and a GaP cap layer above the QD; both thicknesses are roughly equal to three times the QD height.

\section{B. Strain field}

Once the initial supercell is built, the atomic positions are relaxed with a valence force field (VFF) method [41] using two parameters to fit the $\mathrm{C}_{11}$ and $\mathrm{C}_{12}$ macroscopic elastic constants of both $\mathrm{GaAs}$ and GaP [42]. This standard Keating model is known to commit an error on the $\mathrm{C}_{44}$ values and to not properly include the anharmonic effects [43-46]. Nevertheless, for $\mathrm{GaAs}$ and $\mathrm{GaP}$, the error on $\mathrm{C}_{44}$ is only $11 \%$. Moreover in the specific case of [001]-grown QDs [47], the shear strain effects related to the $\mathrm{C}_{44}$ elastic constant are much weaker than the hydrostatic and biaxial strain components. Finally, the error related to the uncertainty on the indium content (see Sec. II) is expected to be larger than the error related to this strain model so that using the standard Keating procedure is relevant in this paper. To model the biaxial strain imposed by the substrate, the two in-plan supercell basis vectors are fixed during the VFF relaxation while the out-of-plan component can relax. Figures 2(a) and 2(b) show the 2D strain mapping for the (001) plan crossing the QD at half of its height. The strain field is found to be quite homogeneous inside the QD. The strain in the GaP matrix tends to zero when going away from the QD (this guarantees that the strain calculation is not affected by boundaries of the supercell). Figure 2(c) shows the strain profile in the $z$ direction (growth direction) on an axis crossing the QD at its center. This profile shows that the biaxial strain is slightly positive in the vicinity of the QD in the GaP barrier, as already demonstrated in Ref. [35].

\section{Electronic eigenstates}

The extended $\mathrm{sp}^{3} \mathrm{~d}^{5} \mathrm{~s}^{*} \mathrm{~TB}$ model, including spin-orbit coupling, is then used to find out the eigenenergies and wave functions of the QD (see Ref. [35] and references therein). This atomistic method is able to describe the electronic band structure throughout the entire Brillouin zone of bulk semiconductors and heterostructures. The TB parameters have been taken from Ref. [48], as they are known to reproduce well the experimental band gaps and the effective masses of the main cubic bulk semiconductors. The Hamiltonian eigenvalues are calculated combining a Lanczos algorithm [49] with a folded spectrum method [50,51].

The TB results for the first four confined hole levels are presented in Table I (each level is doubly degenerated due to spin). The ground hole state is located at $0.458 \mathrm{eV}$ above the
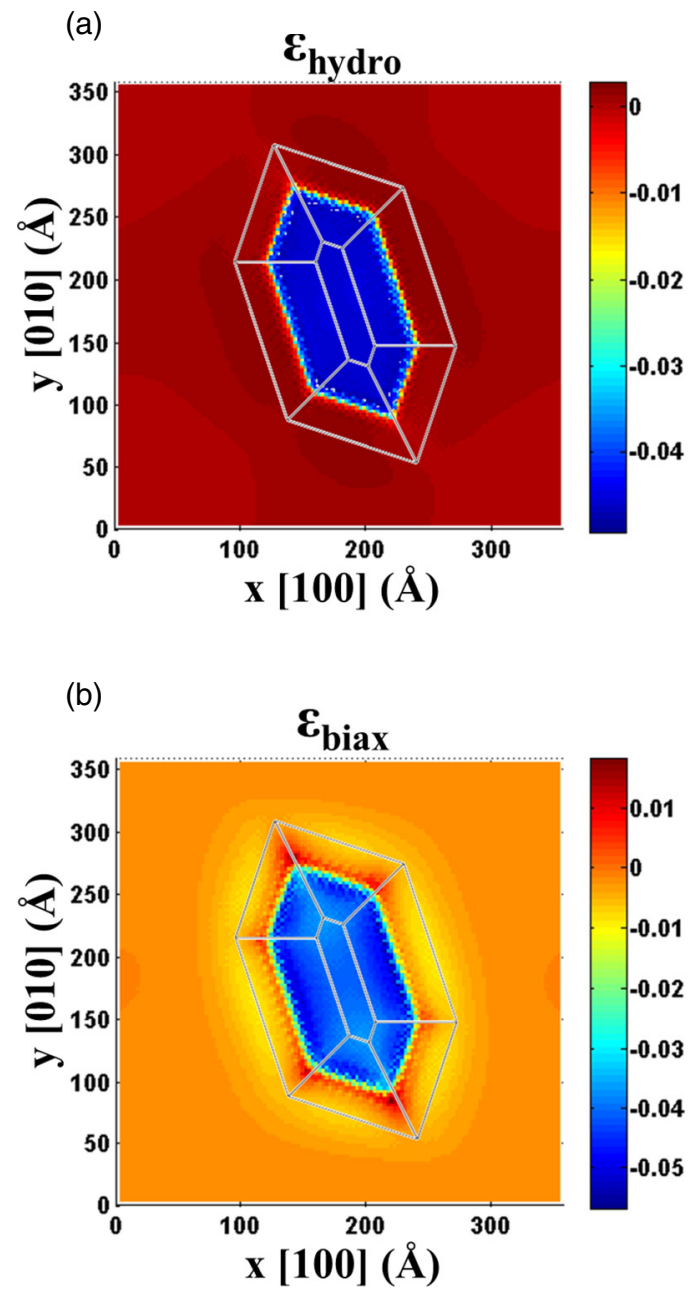

(c)

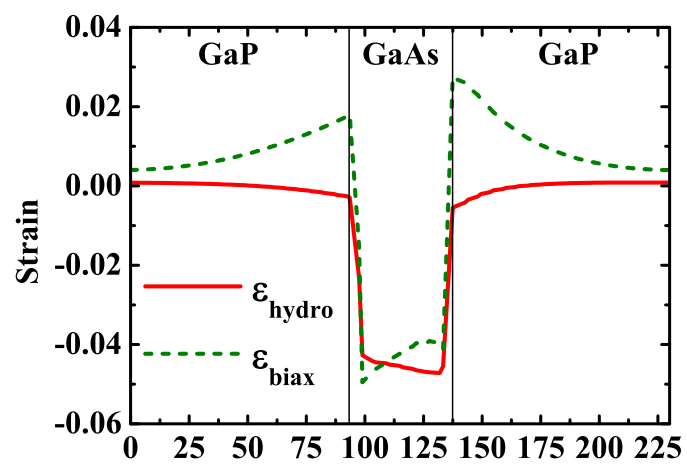

Position along growth direction [001] (§)

FIG. 2. (a), (b) Strain field in a GaAs/GaP QD with hydrostatic $\left(\varepsilon_{\text {hydro }}=\varepsilon_{x x}+\varepsilon_{y y}+\varepsilon_{z z}\right)$ and biaxial $\left(\varepsilon_{\text {biax }}=\frac{1}{2}\left(\varepsilon_{x x}+\varepsilon_{y y}\right)-\right.$ $\left.\varepsilon_{z z}\right)$ components, respectively, represented for the QD midheight plane. (c) Hydrostatic and biaxial strain components along the growth direction [001].

valence band maximum (VBM) of bulk GaP, which is in good agreement with the mixed $\mathrm{k} \cdot \mathrm{p} / \mathrm{TB}$ calculations performed in Ref. [29] in the case of an In-free QD.

The decomposition of the square wave functions (related to electronic density) on the 20 orbitals of the $\mathrm{sp}^{3} \mathrm{~d}^{5} \mathrm{~s}^{*}$ basis 
TABLE I. First four hole states and energies in a GaAs/GaP QD. The reference of energies is the valence band maximum of bulk GaP. The representation of wave functions considers probability volumes including $80 \%$ of the presence probability of the hole. Decomposition is given in terms of the 20 orbitals of the TB basis (spin up and spin down orbitals contribution are added).

\begin{tabular}{|c|c|c|c|}
\hline & Energy $(\mathrm{eV})$ & Hole square wave function & TB orbitals decomposition \\
\hline HH1 & 0.458 & & $\begin{array}{c}s=0.37 \% s^{*}=0.04 \% \\
\boldsymbol{p}_{x}=\mathbf{3 5 . 1 \%} \quad \boldsymbol{p}_{\boldsymbol{y}}=\mathbf{4 0 . 3 \%} \quad p_{z}=3.7 \% \\
d_{y z}=8.95 \% d_{z x}=10.7 \% d_{x y}=0.8 \% \\
d_{x^{2}-y^{2}}=0.004 \% d_{3 z^{2}-r^{2}}=0.009 \%\end{array}$ \\
\hline $\mathrm{HH} 2$ & 0.441 & & $\begin{array}{c}s=0.56 \% s^{*}=0.06 \% \\
\boldsymbol{p}_{x}=\mathbf{3 5 . 3 \%} \quad \boldsymbol{p}_{\boldsymbol{y}}=\mathbf{3 8 . 5 \%} \quad p_{z}=5.5 \% \\
d_{y z}=8.95 \% d_{z x}=10.1 \% d_{x y}=1.2 \% \\
d_{x^{2}-y^{2}}=0.009 \% d_{3 z^{2}-r^{2}}=0.01 \%\end{array}$ \\
\hline HH3 & 0.430 & & $\begin{array}{c}s=0.59 \% s^{*}=0.07 \% \\
\boldsymbol{p}_{x}=\mathbf{3 4 . 8 \%} \quad \boldsymbol{p}_{\boldsymbol{y}}=\mathbf{3 5 . 8 \%} \quad p_{z}=8.7 \% \\
d_{y z}=8.85 \% d_{z x}=9.3 \% d_{x y}=1.9 \% \\
d_{x^{2}-y^{2}}=0.01 \% d_{3 z^{2}-r^{2}}=0.02 \%\end{array}$ \\
\hline HH4 & 0.426 & & $\begin{array}{c}s=0.68 \% s^{*}=0.07 \% \\
\boldsymbol{p}_{\boldsymbol{x}}=\mathbf{3 4 . 5 \%} \boldsymbol{p}_{\boldsymbol{y}}=\mathbf{3 7 . 5 \%} p_{z}=7.3 \% \\
d_{y z}=8.68 \% d_{z x}=9.7 \% d_{x y}=1.6 \% \\
d_{x^{2}-y^{2}}=0.02 \% d_{3 z^{2}-r^{2}}=0.02 \%\end{array}$ \\
\hline
\end{tabular}

is presented in the last column of the table. The main part of the weight is on the $p_{x}$ and $p_{y}$ orbitals for the four hole states calculated here, which proves that they are heavy hole (HH)-like states. The level splitting between the first two levels is only $17 \mathrm{meV}$; this is due to the elongated shape of the QD (with a length of $28 \mathrm{~nm}$ between extreme points at the basis of the QD).

In the same way, we present the results for the different first electron states in Table II. For the ground state $(2.248 \mathrm{eV})$, we find that the electron wave function is not confined in the GaAs QD but outside the QD, close to its top, in the GaP matrix. The main part of the weight is on the $p_{z}, d_{x y}$, and $d_{3 z^{2}-r^{2}}$ orbitals in which the $z$ axis is a symmetry axis. This is characteristic of $X_{Z}$-like states. To confirm this character, we also perform a discrete Fourier transform of each wave function in real space to project it on the k-points of the Brillouin zone of the bulk material. The results are presented in Table III and show the dominant bulk states involved in each confined state [52]. The decomposition of the ground state confirms that it is strongly $X_{Z}$ dominant (more than $95 \%$ ) with a very small fraction coming from the coupling with the $\Gamma$ band $(0.02 \%)$.

This $X_{Z}$-like character was already widely discussed in Ref. [35]. Indeed, it was noticed that the GaP matrix is deformed at the apex of the QD; this results in a positive biaxial strain [see Fig. 2(c)]. The biaxial strain splits the $X$ conduction band into $X_{X Y}$ and $X_{Z}$. In the GaAs QD, the biaxial strain is negative so that the $X_{X Y}$ band is shifted down and the $X_{Z}$ band is shifted up. But in the GaP matrix just above the QD, the situation is reversed with the $X_{Z}$ band being the lowest one. Due to the local strain field, this electron state is located 119 meV below the $X$ band of unstrained GaP.

Now looking at higher electron states of Table II, the next electron excited states are all located within an interval of $40 \mathrm{meV}$ (from 2.26 to $2.30 \mathrm{eV}$ ). As illustrated by the decomposition of the first excited state $(2.262 \mathrm{eV})$ of Table II and Table III, these are all $X_{Z}$-like states mechanically confined above and even below the GaAs QD. This result is in good agreement with strain calculations performed in Fig. 2(c), where it was noticed that the biaxial strain is slightly positive in the GaP barrier at the apex and below the QD, which explains the localization of electrons in these particular areas corresponding to $X_{Z}$ confinement potentials. Nevertheless, the confinement potential below the QD is weaker than the one above the QD and explains why the states confined below the QD are at a higher energy than the states confined above it. The first electron state confined in the QD is finally found at the energy of $2.31 \mathrm{eV}$ (see Table II) that is $62 \mathrm{meV}$ above the ground state and $57 \mathrm{meV}$ below the $X$ band of unstrained bulk $\mathrm{GaP}$. This is a $X_{Y}$-like state as proved by the strong weight on the $p_{y}$ orbital and by the result of the Fourier decomposition of Table III. Other $X_{X}$ and $X_{Y}$-like states are found at energies below the $X$ band of unstrained bulk GaP barrier. In contrast, no $\Gamma$-like confined state is found in this paper because it lies well above the $X$ band of unstrained bulk GaP barrier (for a 
TABLE II. Electron eigenstates and energies in a GaAs/GaP QD. The table describes the two lower energy states (at 2.248 and $2.262 \mathrm{eV}$ ), excited states in the [2.268-2.306] eV range, and the first electron state confined in the QD (at $2.310 \mathrm{eV}$ ). The reference of energies is the valence band maximum of bulk GaP. The representation of wave functions considers probability volumes including $80 \%$ of the presence probability of the electron. Decomposition is given in terms of the 20 orbitals of the TB basis (spin up and spin down orbitals contribution are added).

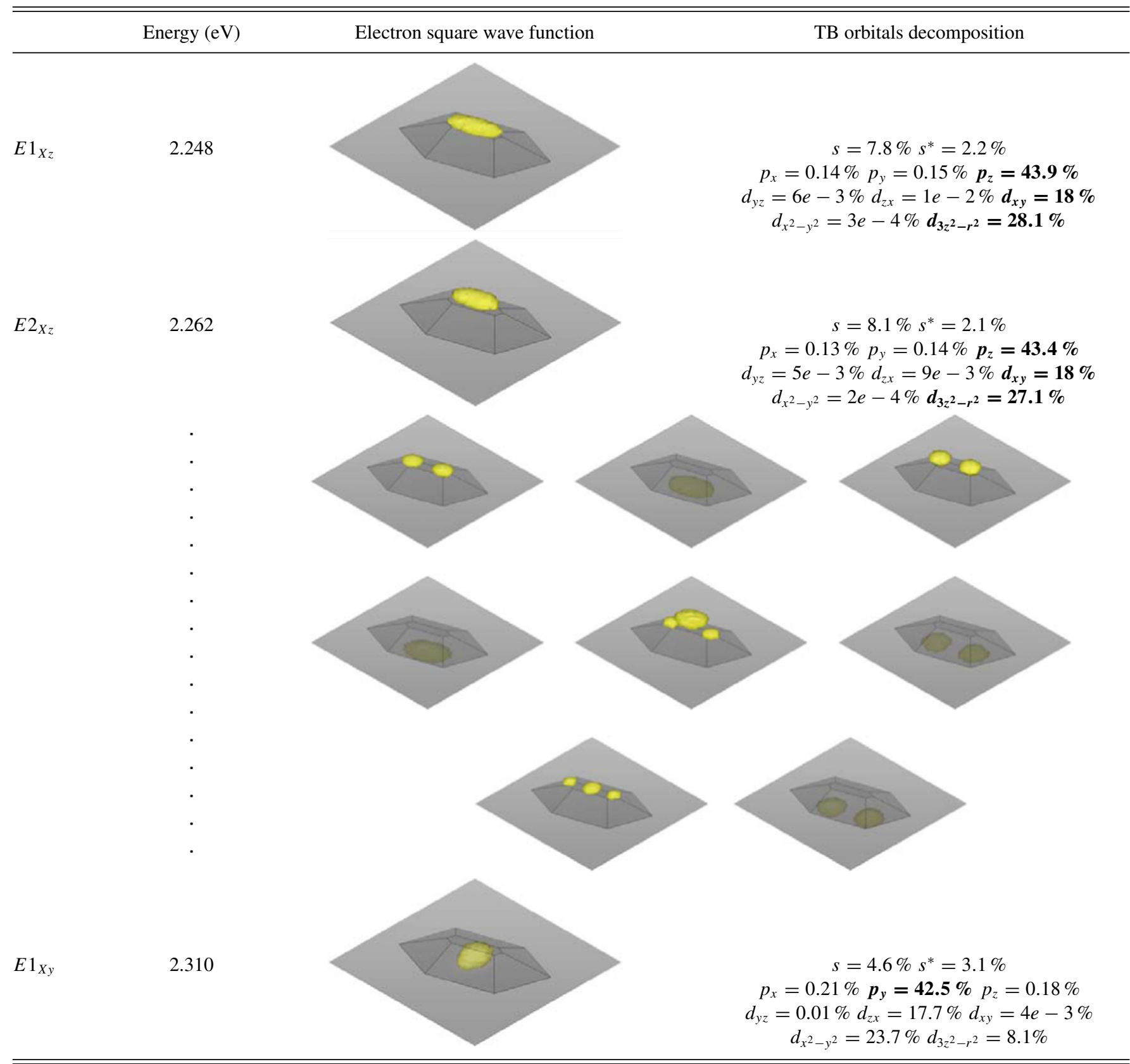

$0 \%$ In content, here). In conclusion, according to the supercell TB simulation of a GaAs/GaP QD, the $X_{X Y}$ state of QD is actually not the electronic ground state. Many electron states mechanically confined at the apex and at the base of the QD are found at lower energies. We can also predict that increasing slightly the In content inside the QD would not affect the presence of these states. Indeed, increasing the In content increases the lattice mismatch between the dot and the GaP matrix. Consequently, the tensile strain in the GaP matrix at the apex of the dot increases with In content and so does the confinement potential for the $X_{Z}$ electrons. We now present the role of these electron states on the optical transitions with the first confined hole states.

\section{Calculated absorption spectrum}

Optical properties are derived from the dimensionless oscillator strength calculation given as a function of the direction of electric field by

$$
f(\hat{e})=\frac{2}{m_{0}} \frac{\left|\hat{e} \cdot \boldsymbol{p}_{v c}(\boldsymbol{k})\right|^{2}}{\left(E_{c}(\boldsymbol{k})-E_{v}(\boldsymbol{k})\right)},
$$


TABLE III. Decomposition of the electron confined states on the bulk states.

\begin{tabular}{lclllll}
\hline \hline & Energy $(\mathrm{eV})$ & $X_{X}$ & $X_{Y}$ & $X_{Z}$ & $L$ & $\Gamma$ \\
\hline$E 1_{X z}$ & 2.248 & 0.00000 & 0.00000 & $\mathbf{0 . 9 5 1 2 5}$ & 0.00001 & 0.00021 \\
$E 2_{X z}$ & 2.262 & 0.00000 & 0.00000 & $\mathbf{0 . 9 8 8 9 9}$ & 0.00001 & 0.00031 \\
$E 3_{X z}$ & 2.267 & 0.00000 & 0.00000 & $\mathbf{0 . 9 4 7 9 8}$ & 0.00001 & 0.00023 \\
$E 4_{X z}$ & 2.281 & 0.00000 & 0.00000 & $\mathbf{0 . 9 7 6 7 4}$ & 0.00001 & 0.00011 \\
$E 5_{X z}$ & 2.283 & 0.00000 & 0.00000 & $\mathbf{0 . 9 8 8 6 8}$ & 0.00002 & 0.00030 \\
$E 6_{X z}$ & 2.287 & 0.00000 & 0.00000 & $\mathbf{0 . 9 9 4 7 7}$ & 0.00000 & 0.00016 \\
$E 7_{X z}$ & 2.294 & 0.00000 & 0.00000 & $\mathbf{0 . 9 4 8 4 5}$ & 0.00001 & 0.00017 \\
$E 8_{X z}$ & 2.296 & 0.00000 & 0.00000 & $\mathbf{0 . 9 7 4 8 3}$ & 0.00000 & 0.00012 \\
$E 9_{X z}$ & 2.299 & 0.00000 & 0.00000 & $\mathbf{0 . 9 4 9 6 7}$ & 0.00001 & 0.00015 \\
$E 10_{X z}$ & 2.303 & 0.00000 & 0.00000 & $\mathbf{0 . 9 9 4 4 9}$ & 0.00000 & 0.00017 \\
$E 1_{X y}$ & 2.310 & 0.00000 & $\mathbf{1 . 0 0 0 0 0}$ & 0.00000 & 0.00000 & 0.00000 \\
\hline \hline
\end{tabular}

where $\hat{e}$ is a unit vector in the direction of the optical electric field, $E_{v}(\boldsymbol{k})$ and $E_{c}(\boldsymbol{k})$ are the energies of the initial and final states, $m_{0}$ is the reduced effective mass, and $\boldsymbol{p}_{v c}(\boldsymbol{k})$ are the momentum matrix elements that are calculated using a $k$-derivation of the TB-Hamiltonian [53]. The concept of absorption in $\mathrm{cm}^{-1}$ for a QD system can only be defined when considering the volume density of QD. For a QD, we prefer to give it in arbitrary units, such as in Fig. 3(a), or calculate the radiative lifetime for a given optical transition [54]:

$$
\tau_{\mathrm{rad}}=\frac{2 \pi \varepsilon_{0} m_{0} c^{3} \hbar^{2}}{n_{r} e^{2}\left(E_{c}-E_{v}\right)^{2} f_{\mathrm{eff}}} .
$$

Here $f_{\text {eff }}$ is the effective oscillator strength, which is calculated by integrating the oscillator strength $f(\hat{e})$ over the unit sphere

$$
f_{\text {eff }}=\int_{0}^{\pi} \int_{0}^{2 \pi} f(\hat{e}) \sin \varphi d \theta d \varphi
$$

and $n_{r}$ is the effective refractive index taking into account the difference between the refractive indexes of both QD and barrier material [55],

$$
n_{r}=\frac{9 n_{\text {barrier }}^{5}}{\left(2 n_{\text {barrier }}^{2}+n_{\mathrm{QD}}^{2}\right)^{2}} .
$$

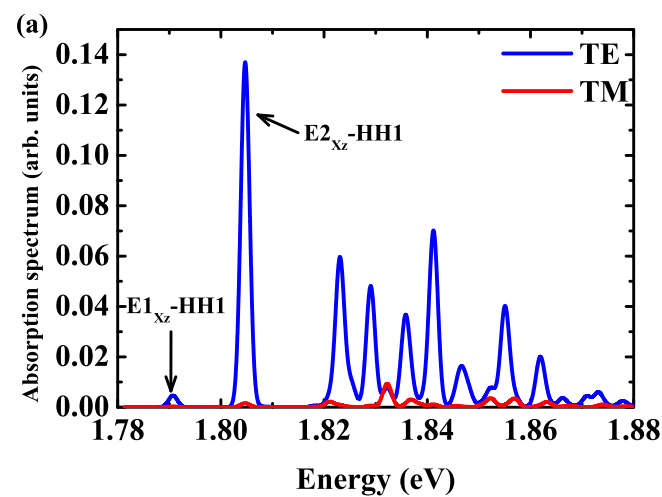

Figure 3(a) presents the calculation of the absorption spectrum for transverse electric (TE) or transverse magnetic (TM) polarizations of the incoming light. The first four hole states (each being doubly degenerated due to spin) and the first 13 electron states (each being doubly degenerated due to spin) are considered in this calculation. A first conclusion is that the TE polarization is strongly dominant. This is a consequence of optical transitions involving $\mathrm{HH}$ states. We also notice the numerous peaks above $1.82 \mathrm{eV}$, which are the transitions involving the many electron states of Table II. Interestingly, the second optical transition $E 2_{X z}-\mathrm{HH} 1$ at $1.804 \mathrm{eV}$ is found to be more efficient than the ground one $E 1_{X z}-\mathrm{HH} 1$ at $1.79 \mathrm{eV}$. This may be explained by two reasons: (i) a strongest spatial overlap due to a slightly stronger electron wave function penetration in the QD or (ii) a slightly stronger contribution of the $\Gamma$ bulk states, as proved by the results of Table III. The calculated corresponding radiative lifetime is $9.6 \mu \mathrm{s}$. Despite the favored spatial overlap, the first type-I optical transition $\left(E 1_{X y}-\mathrm{HH} 1\right)$ is much less efficient. The calculated radiative lifetime is $2.4 \mathrm{~s}$. This may be a consequence of the absence of $\Gamma$ bulk state contribution in this electron confined state, as shown in Table III.

\section{OPTICAL PROPERTIES}

\section{A. Radiative lifetimes}

The carrier dynamics for low In content (In,Ga)As/GaP QDs was studied earlier at short time ranges $(<12 \mathrm{~ns})$ by using time-resolved PL [29]. While providing a good description of the carrier dynamic below the ns range, the experimental conditions were not suitable to measure long radiative lifetimes. In this paper, a sample, including one (In,Ga)As/GaP QDs single layer grown on GaP substrate by gas-source molecular beam epitaxy with In content typically below 15\% (see the growth conditions in Ref. [20]), is excited by a frequency-doubled Ti:sapphire laser at the wavelength of $405 \mathrm{~nm}$ with a pulse picker, providing a repetition rate as small as $0.8 \mathrm{MHz}$. The PL signal is analyzed by a S20 streak camera with a time resolution of $\sim 250 \mathrm{ps}$, and measurements are performed at $10 \mathrm{~K}$. The measured excitation density is $\sim 0.6 \mathrm{~W} \mathrm{~cm}^{-2}$. The decay, shown in Fig. 3(b), can be described by a mono exponential, indicating no Auger effects at this low excitation power. From these measurements, the carrier

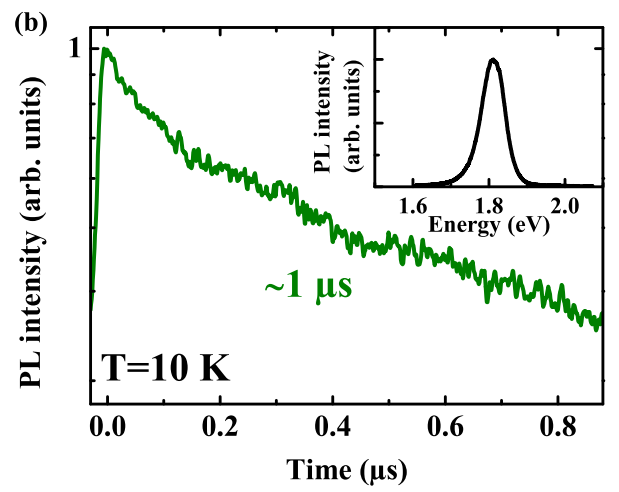

FIG. 3. (a) Absorption spectrum of the GaAs/GaP QD calculated with the TB supercell model. (b) Normalized PL dynamic at $10 \mathrm{~K}$ of $(\mathrm{In}, \mathrm{Ga}) \mathrm{As} / \mathrm{GaP} \mathrm{QDs}$ with a low In content. The repetition rate is $0.8 \mathrm{MHz}$, and the excitation density is $0.6 \mathrm{~W} . \mathrm{cm}^{-2}$. The inset shows the time integrated spectrum. 
lifetime inside the QDs is estimated around $1 \mu \mathrm{s}$. This result is of the same order of magnitude than the radiative lifetime calculated with the TB model in the previous section, for the $E 2_{X z}-\mathrm{HH} 1$ transition $(9.6 \mu \mathrm{s})$. The discrepancy may be explained by the fact that we simulated a pure $\mathrm{GaAs} / \mathrm{GaP}$ QD instead of a low In-content InGaAs/GaP QD. Indeed, Prohl et al. [30] reported a reversed cone stoichiometry in their $\mathrm{In}_{0.25} \mathrm{Ga}_{0.75} \mathrm{As} / \mathrm{GaP} \mathrm{QD}$, which should lead to a spatial localization of the hole wave function closer to the top of the QD, enhancing the spatial overlap with the electron wave function. Second, we cannot exclude for such a long decay time that some nonradiative recombination channels also play a role even at $10 \mathrm{~K}$.

\section{B. Effect of the hydrostatic pressure}

Strain is known to have a deep impact on the band structure of semiconductors. Pressure dependent PL is thus a convenient technique to probe the nature of the optical transitions in QDs [56,57]. In this paper, the same sample as in Sec. III.A is mechanically thinned to a thickness of approximately $30 \mu \mathrm{m}$ and then cut into pieces of about $100 \times 100 \mu \mathrm{m}^{2}$ in size. The PL is excited using the $405 \mathrm{~nm}$ line of a continuous wave laser diode, and the PL signal is collected using a LabRam HR800 spectrometer equipped with a charge-coupled device detector. High pressure experiments are performed at room temperature, employing a gasketed diamond anvil cell to generate hydrostatic pressures up to $10 \mathrm{GPa}$ [56]. A 4:1 methanol:ethanol mixture is used as a pressure transmitting medium. Pressure is measured using the ruby fluorescence method [58].

Figure 4(a) shows the PL spectra obtained for various values of hydrostatic pressure. The raw data are represented by thin lines. The interference fringes due to multiple reflections of the emitted light between both plane parallel facets of the polished sample are readily observed. The double, very sharp peaks at around $1.78 \mathrm{eV}$ correspond to the ruby luminescence used to determine the pressure. The thick lines show smoothed spectra. The thin black line is a guide to the eye, corresponding to the energy of the maximum PL intensity, which is plotted in Fig. 4(b) as solid symbols.

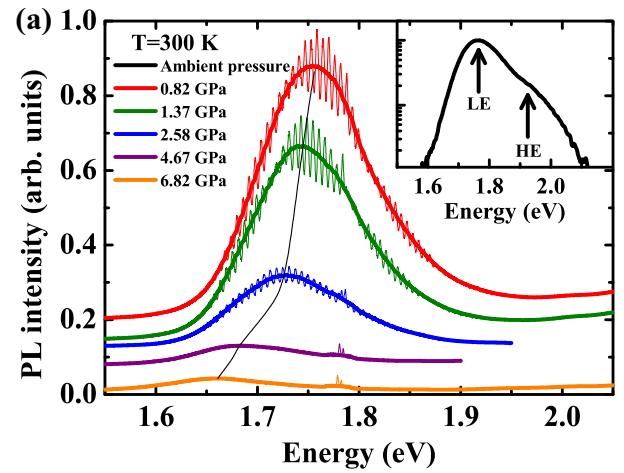

We first notice the monotonic redshift of the PL peak with increasing pressure. This feature can be understood by considering the effect of a hydrostatic compressive stress on the $\Gamma, X$, and $L$ conduction band minima. The direct $\Gamma-\Gamma$ band gap and indirect $L-\Gamma$ band gap both blueshift with increasing pressure, whereas the indirect $X-\Gamma$ gap decreases with increasing pressure [59]. Hence, the observed redshift unambiguously proves that the PL peak involves $X$-type conduction states in good correlation with TB calculations. This was already discussed in detail in a previous work [35], in which it was noticed that the measured linear variation roughly corresponds to the expected pressure dependence for an $X$-like level in $\mathrm{GaP}\left(-13 \mathrm{meV} \mathrm{GPa}^{-1}\right.$ : the measured pressure coefficient of the indirect $X-\Gamma$ bandgap in bulk GaP [60]).

We now analyze in more detail the high pressure results by carefully inspecting the PL lineshape and its changes under pressure to show that the broad PL peak is actually composed of several optical transitions. At ambient pressure [see inset of Fig. 4(a) and Ref. [29]], two peaks of LE and high energy (HE), separated from the LE peak by $100-150 \mathrm{meV}$, have been clearly identified. Here, the HE transition is no more observed as soon as the pressure is applied. An explanation can be proposed based on our assumption of a HE transition of direct type [29]. Indeed, a direct $\Gamma-\Gamma$ transition exhibits a large positive pressure coefficient (for bulk GaAs, the pressure coefficient of the direct band gap is around $110 \mathrm{meV} \mathrm{GPa}^{-1}$ ) [61]. As soon as a slight hydrostatic pressure is applied, the HE transition (if assumed to be a direct transition) would strongly shift up in energy, while the LE transition (which is an indirect transition involving $X$ conduction band states) shifts down in energy. The intensity ratio between the two transitions follows an exponential law of the level splitting; therefore, it is then not surprising to quench the $\mathrm{HE}$ transition for the smallest pressure $(0.82 \mathrm{GPa})$ considered in this paper. Another reason is the shift down in energy of the $X$ conduction state of GaP barrier with increasing pressure. When the $\Gamma$ electronic state of the QDs goes above the $X$ state of the GaP barrier, the probability of filling the $\Gamma$ electronic states of the QDs is further strongly reduced.

Although the direct transition is not observed in the pressure dependent PL spectra, we have solid hints to claim that the

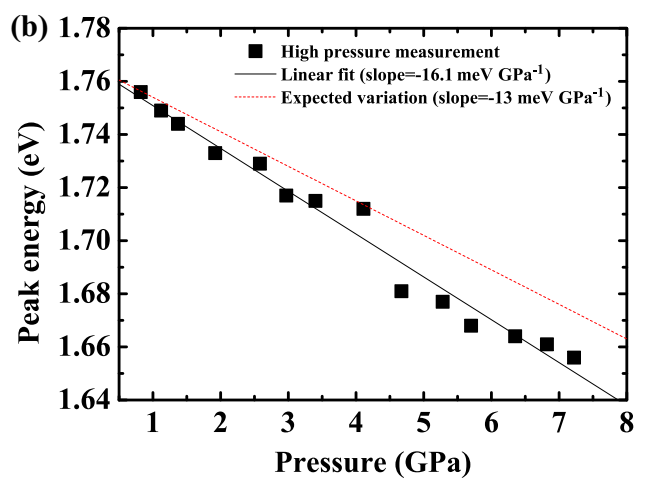

FIG. 4. (a) The PL spectrum of low In content (In,Ga)As/GaP QDs at room temperature as a function of hydrostatic pressure. The raw data are shown by thin lines. The spectra are smoothed (thick lines) to avoid the interference fringes. The thin black line is a guide to the eye to highlight the position of the PL maximum. The inset shows the PL spectrum in ambient pressure conditions (outside the diamond anvil cell) in logarithmic scale. The LE and HE peaks are marked by arrows. (b) Variation of the energy of the maximum PL intensity $E_{\max }$ as a function of pressure. The thin red dashed line shows an expected linear variation with a pressure coefficient of $-13 \mathrm{meV} \mathrm{GPa}^{-1}$. The thin black line shows the linear fit result with a slope of $-16.1 \mathrm{meV} \mathrm{GPa}^{-1}$. 
(a)

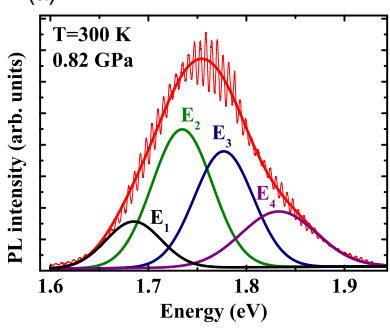

(b)

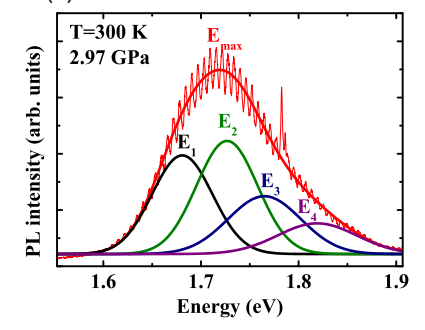

FIG. 5. The PL spectrum of low In content (In,Ga)As/GaP QDs at (a) $0.82 \mathrm{GPa}$ and (b) $2.97 \mathrm{GPa}$ and fitting with four Gaussian peaks centered at $E_{1}, E_{2}, E_{3}$, and $E_{4}$. (c) Pressure dependence of the energies $E_{\max }$ of the maximum PL intensity and of the four Gaussian peaks used to fit the spectra. The FWHM of the four Gaussian peaks are kept constant as a function of pressure.

peak is composed of several optical transitions. The first one relies on the redshift of the energy of the PL maximum $E_{\max }$ [see Fig. 4(b)]. The linear fit performed on the data [thin black line in Fig. 4(b)] shows that the PL maximum red shifts with pressure at a rate of $-16.1(6) \mathrm{meV} \mathrm{GPa}^{-1}$. This is thus stronger than $-13 \mathrm{meV} \mathrm{GPa}^{-1}$, the pressure coefficient we could have expected in first approximation and corresponding to the pressure coefficient of the indirect $X$ - $\Gamma$ bandgap in bulk GaP [60] [thin red line in Fig. 4(b)]. As shown below, a larger pressure coefficient than that of the bulk is not consistent with the overall changes in the energetics and the variation of the built-in strain of the QD structure induced by the hydrostatic pressure. Second, the full width at half maximum (FWHM) of the PL peak is larger than $100 \mathrm{meV}$, a result which is not consistent with the expected width of the QD emission due to inhomogeneous broadening. A peak width of about $50 \mathrm{meV}$ can be inferred from the dot-size fluctuations reported here, in agreement with observations made in similar dot systems [46]. A careful analysis of the PL lineshape indicates that the emission spectrum actually consists of a superposition of inhomogeneously broadened Gaussian peaks, although large widths prevent us from resolving individual peaks. Figures 5(a) and 5(b) show two examples of the lineshape analysis performed with four Gaussians used to fit the PL spectrum at 0.82 and $2.97 \mathrm{GPa}$, respectively. Starting with the lowest energy peak, each one is labeled as $E_{i}$ with $i=1,2, \ldots$, corresponding to the principal quantum number due to confinement. Given the TB results of Table II, we attribute these peaks to the various optical transitions involving the ground hole state confined into the dots and the $X_{Z}$-like states confined at the dot apex $\left(E 1_{X z}-\mathrm{HH} 1, E 2_{X z}-\mathrm{HH} 1, \ldots\right)$. We emphasize that four is the minimum number of Gaussians that allows for a very satisfactory description of the PL line shape [red line in Figs. 5(a) and 5(b)] and its changes upon the application of external pressure. This cannot be achieved with less peaks, and, in turn, an additional one is just redundant. We note that the FWHM of the four Gaussian peaks are kept constant as a function of pressure since the inhomogeneous broadening is not expected to change under pressure.

In Fig. 5(c), the energies of the four Gaussian peaks are plotted as a function of pressure (full symbols) and compared to the energy $E_{\max }$ of the maximum PL intensity (red open squares). The pressure dependences of $E_{1}, E_{2}, E_{3}$, and $E_{4}$ are linear, with pressure coefficients ranging roughly between $-1 \mathrm{meV} \mathrm{GPa}^{-1}$ and $-5 \mathrm{meV} \mathrm{GPa}^{-1}$. The negative values ensure that these transitions occur from $X$-type conduction band minima. Moreover, depending on pressure, $E_{\max }$ coincides with the points at which a different Gaussian peak is the dominant one. For example, at $0.82 \mathrm{GPa}$ [Fig. 5(a)], both peaks $E_{2}$ and $E_{3}$ are almost equally intense, but at $2.97 \mathrm{GPa}$ [Fig. 5(b)], the $E_{1}$ and $E_{2}$ peaks have the highest intensity. When pressure is increased above $4.5 \mathrm{GPa}$, the $E_{1}$ peak becomes the dominant one. These changes in relative intensities are at the origin of the apparently strong and partially stepwise variation of the energy of the PL maximum with pressure, as illustrated in Fig. 5(c).

We now turn to the discussion of the magnitude of the negative pressure coefficients of the $E_{1}, \ldots, E_{4}$ transition energies, which are much smaller than $-13 \mathrm{meV} \mathrm{GPa}^{-1}$, the value measured for bulk GaP [60]. The situation is completely similar to that found for the InP/GaP QD system [46], where a similar reduction was observed. The reason for such a behavior is the gradual decrease of the built-in strain (tensile or compressive) present in the structure with increasing hydrostatic pressure. The residual strain is a consequence of the lattice mismatch between dot and barrier material, InGaAs and $\mathrm{GaP}$, in this case, respectively. Since the compressibility of $\mathrm{GaP}$ is smaller than the one of InGaAs, the lattice mismatch between both materials reduces under application of an external hydrostatic pressure and so does the built-in strain. In our particular case, this means that the splitting of the $X$ valleys of $\mathrm{GaP}$ in the region, where the residual tensile strain produces the confinement of the electrons giving rise to the $E_{1}, \ldots, E_{4}$ transitions, also decreases with increasing pressure. Consequently the shift down of the $X_{Z}$ states would be smaller than $13 \mathrm{meV} \mathrm{GPa}^{-1}$ in absolute value because the contribution of the $X$ valley splitting reduction acts against the redshift of the "centroid" of the $X$ valleys. This situation is sketched in Fig. 6.

Another somewhat intriguing point is why the magnitude of the pressure coefficient is systematically smaller, the higher the transition energy is, or, in other words, the higher the confinement energy of the $X_{Z}$ electrons. Here we can only speculate, arguing as in the case of the pressure dependence of optical transitions in amorphous Si nanoparticles embedded in a substoichiometric oxide matrix [62]. In this paper, it was shown by combining high-pressure PL experiments and ab-initio band structure calculations that the sign and magnitude of the pressure derivative of transition energies from different electron states are solely determined by the confinement energy. In the case of the Si nanoparticles, it was argued that with increasing confinement energy, the bulk states contributing the most to the confinement-level wave function stem from regions in reciprocal space that are further 


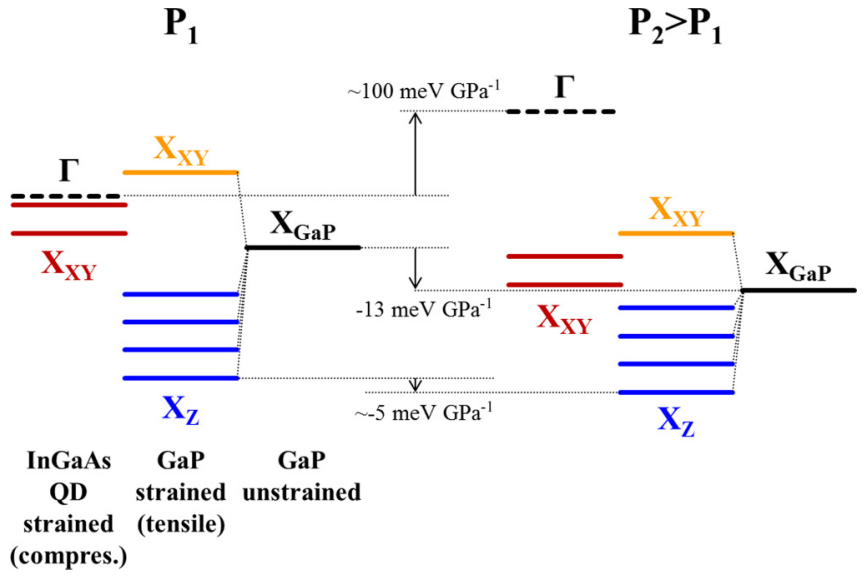

FIG. 6. Sketch of the effect of applied pressure on the $X$ band of $\mathrm{GaP}$ barrier and $X_{X Y}$ and $X_{Z}$ states of (In)GaAs QD.

away from the $X$ point, having less negative or even positive deformation potentials, as compared to the states at the $X$ point. For the InGaAs/GaP QDs, however, the decomposition in terms of the bulk states given in Table III does not support the interpretation of the observed systematics in the pressure coefficients in these terms. The reason for that remains elusive.

A striking result that gives further support to the proposed interpretation concerns the peculiar pressure dependence of the peak intensities, as depicted in Fig. 7. Since the intensities are plotted in logarithmic scale, the data of Fig. 7 clearly indicate that the peak intensities decrease exponentially with increasing pressure. Furthermore, the decay is more pronounced for higher transition energies. In the present case, the excitation of the PL proceeds at an energy above the gap of GaP, the barrier materials. In this way, most of the photoexcited carriers are generated inside the barriers, from which they are subsequently captured either by the QDs (holes) or the potential well at the tensily strained $\mathrm{GaP}$ regions (electrons), to finally recombine radiatively. Obviously, at room temperature there is a finite probability that carriers will escape (principally electrons due to the extremely large barrier height for holes) from the confining region back into the unstrained regions of the GaP barriers. As becomes clear from the sketches of Fig. 6, the escape barrier for electrons is pressure dependent not only

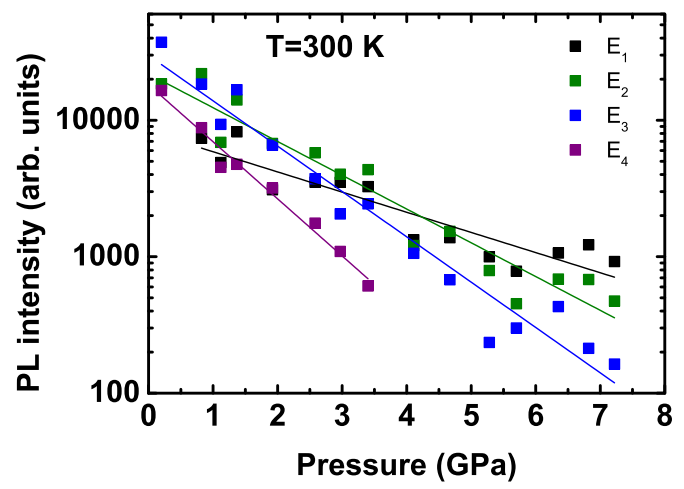

FIG. 7. Intensity (in logarithmic scale) of each PL Gaussian peak with energy $E_{1}$ to $E_{4}$ as a function of pressure (closed symbols). The solid lines correspond to linear fits to the data points using Eq. (3). because of the different pressure coefficient for $E_{1}, \ldots, E_{4}$ but also because the barrier itself is pressure dependent,

$$
E_{b, i}=E_{\mathrm{GaP}}^{X}-E_{i}=E_{b, i}^{0}-a_{i} \times P \quad i=1, \ldots, 4,
$$

where $E_{b, i}^{0}$ is the barrier height at ambient pressure and $a_{i}$ is its pressure coefficient,

$$
a_{i}=d E_{i} / d P-d E_{\mathrm{GaP}}^{X} / d P \quad i=1, \ldots, 4,
$$

where $d E_{\mathrm{GaP}}^{X} / d P=-13 \mathrm{meV} \mathrm{GPa}^{-1}$ is the pressure coefficient of the indirect $X-\Gamma$ gap of GaP. In Fig. 5(c), we notice that the derivative $d E_{i} / d P$ for each transition energy $E_{i}$ is smaller in absolute value for higher $E_{i}$. This implies that the pressure derivative $a_{i}$ of the barrier height is stronger for higher $E_{i}$. In addition, solving the rate equations for a simplified model in which a constant carrier-capture probability is assumed, whereas the escape probability depends exponentially on the barrier height, yields the following relationship between peak intensity and pressure:

$$
\operatorname{Ln}\left(I_{i}\right)=-a_{i} / k_{B} T \times P+\text { const. }
$$

The results shown in Fig. 7 speak for the correctness of this relation. The higher the $E_{i}$, the steeper is the intensity reduction, which is a direct consequence of a stronger escape rate of electrons from the regions of mechanical confinement into the unstrained regions of the $\mathrm{GaP}$ barriers. In fact, the values of $a_{i}$ for the pressure derivative of the escape barrier height calculated either from Eq. (6), i.e., from the pressure coefficients of the energies $E_{i}$, or from Eq. (7), that is the slopes of the intensity plots of Fig. 7, agree within $10 \%$ at least for the first two confined levels $E_{1}$ and $E_{2}$.

\section{CONCLUSION}

To conclude, we have unraveled the complex structure of low In-content InGaAs/GaP QD. Particularly, we have demonstrated theoretically and experimentally that several indirect optical transitions are involved in the room temperature PL spectrum. Supercell TB simulations show that these transitions are between electrons with $X_{Z}$ character confined by the strain field in the GaP barrier mainly at the apex of the QD and the first hole state confined inside the dot. The calculation of the radiative lifetime of the most efficient transition is in good agreement with the decay time measured in tr-PL. The measurements of PL with hydrostatic pressure are also in good agreement with the scheme calculated by the TB model.

\section{ACKNOWLEDGMENTS}

A.R.G. and M.I.A. acknowledge support from the Spanish Ministry of Economy and Competitiveness (MINECO) through Grant No. MAT2009-09480 (PIEZOHM) and Severo Ochoa Excellence Centre Award (No. SEV-2015-0496). M.O.N. acknowledges financial support from the Russian Foundation for Basic Research. This research is supported by "Région Bretagne" through the PONANT project including FEDER funds. This paper is also supported by the OPTOSI Agence Nationale pour la Recherche Project No. 12-BS03002-02. This work has been performed using HPC resources of GENCI CINES, TGCC/CCRT, and IDRIS under the allocation No. 2013-[x2013096724]. 
[1] D. Liang and J. E. Bowers, Nat. Photon. 4, 511 (2010).

[2] A. W. Fang, H. Park, O. Cohen, R. Jones, M. J. Paniccia, and J. E. Bowers, Opt. Express 14, 9203 (2006).

[3] X. Sheng, C. Robert, S. Wang, G. Pakeltis, B. Corbett, and J. A. Rogers, Laser Photonics Rev. 9, L17 (2015).

[4] L. Cerutti, J. B. Rodriguez, and E. Tournie, IEEE Photonics Technol. Lett. 22, 553 (2010).

[5] A. Y. Liu, C. Zhang, J. Norman, A. Snyder, D. Lubyshev, J. M. Fastenau, A. W. K. Liu, A. C. Gossard, and J. E. Bowers, Appl. Phys. Lett. 104, 041104 (2014).

[6] T. Wang, H. Liu, A. Lee, F. Pozzi, and A. Seeds, Opt. Express 19, 11381 (2011).

[7] K. Volz, A. Beyer, W. Witte, J. Ohlmann, I. Németh, B. Kunert, and W. Stolz, J. Cryst. Growth 315, 37 (2011).

[8] K. Yamane, K. Noguchi, S. Tanaka, Y. Furukawa, H. Okada, H. Yonezu, and A. Wakahara, Appl. Phys. Express 3, 074201 (2010)

[9] T. Quinci, J. Kuyyalil, T. N. Thanh, Y. P. Wang, S. Almosni, A. Létoublon, T. Rohel, K. Tavernier, N. Chevalier, O. Dehaese, N. Boudet, J. F. Bérar, S. Loualiche, J. Even, N. Bertru, A. L. Corre, O. Durand, and C. Cornet, J. Cryst. Growth 380, 157 (2013).

[10] T. J. Grassman, J. A. Carlin, B. Galiana, L.-M. Yang, F. Yang, M. J. Mills, and S. A. Ringel, Appl. Phys. Lett. 102, 142102 (2013).

[11] A. C. Lin, M. M. Fejer, and J. S. Harris, J. Cryst. Growth 363, 258 (2013).

[12] Y. Ping Wang, A. Letoublon, T. Nguyen Thanh, M. Bahri, L. Largeau, G. Patriarche, C. Cornet, N. Bertru, A. Le Corre, and O. Durand, J. Appl. Crystallogr. 48, 702 (2015).

[13] Y. P. Wang, J. Stodolna, M. Bahri, J. Kuyyalil, T. N. Thanh, S. Almosni, R. Bernard, R. Tremblay, M. D. Silva, A. Létoublon, T. Rohel, K. Tavernier, L. Largeau, G. Patriarche, A. L. Corre, A. Ponchet, C. Magen, C. Cornet, and O. Durand, Appl. Phys. Lett. 107, 191603 (2015).

[14] C. Robert, A. Bondi, T. Nguyen Thanh, J. Even, C. Cornet, O. Durand, J. P. Burin, J. M. Jancu, W. Guo, A. Létoublon, H. Folliot, S. Boyer-Richard, M. Perrin, N. Chevalier, O. Dehaese, K. Tavernier, S. Loualiche, and A. Le Corre, Appl. Phys. Lett. 98, 251110 (2011).

[15] C. Robert, M. Perrin, C. Cornet, J. Even, and J. M. Jancu, Appl. Phys. Lett. 100, 111901 (2012).

[16] K. Jandieri, M. K. Shakfa, S. Liebich, M. Zimprich, B. Kunert, C. Karcher, A. Chernikov, K. Volz, W. Stolz, M. Koch, S. Chatterjee, W. Heimbrodt, F. Gebhard, and S. D. Baranovskii, Phys. Rev. B 86, 125318 (2012).

[17] C. Karcher, K. Jandieri, B. Kunert, R. Fritz, M. Zimprich, K. Volz, W. Stolz, F. Gebhard, S. D. Baranovskii, and W. Heimbrodt, Phys. Rev. B 82, 245309 (2010).

[18] S. Liebich, M. Zimprich, A. Beyer, C. Lange, D. J. Franzbach, S. Chatterjee, N. Hossain, S. J. Sweeney, K. Volz, B. Kunert, and W. Stolz, Appl. Phys. Lett. 99, 071109 (2011).

[19] S.-H. Park, J. Appl. Phys. 115, 063101 (2014).

[20] T. Nguyen Thanh, C. Robert, C. Cornet, M. Perrin, J. M. Jancu, N. Bertru, J. Even, N. Chevalier, H. Folliot, O. Durand, and A. Le Corre, Appl. Phys. Lett. 99, 143123 (2011).

[21] K. Rivoire, S. Buckley, Y. Song, M. L. Lee, and J. Vučković, Phys. Rev. B 85, 045319 (2012).

[22] L. Bonato, E. M. Sala, G. Stracke, T. Nowozin, A. Strittmatter, M. N. Ajour, K. Daqrouq, and D. Bimberg, Appl. Phys. Lett. 106, 042102 (2015)
[23] Y. Song and M. L. Lee, Appl. Phys. Lett. 100, 251904 (2012).

[24] Y. Song and M. L. Lee, Appl. Phys. Lett. 103, 141906 (2013).

[25] M. Heidemann, S. Höfling, and M. Kamp, Appl. Phys. Lett. 104, 011113 (2014).

[26] J.-P. Gauthier, C. Robert, S. Almosni, Y. Léger, M. Perrin, J. Even, A. Balocchi, H. Carrère, X. Marie, C. Cornet, and O. Durand, Appl. Phys. Lett. 105, 243111 (2014).

[27] C. Robert, T. Thanh, C. Cornet, P. Turban, M. Perrin, A. Balocchi, H. Folliot, N. Bertru, L. Pedesseau, M. Nestoklon, J. Even, J.-M. Jancu, S. Tricot, O. Durand, X. Marie, and A. L. Corre, Nanoscale Res. Lett. 7, 643 (2012).

[28] C. Robert, T. N. Thanh, A. Létoublon, M. Perrin, C. Cornet, C. Levallois, J. M. Jancu, J. Even, P. Turban, A. Balocchi, X. Marie, O. Durand, and A. Le Corre, Thin Solid Films 541, 87 (2013).

[29] C. Robert, C. Cornet, P. Turban, T. Nguyen Thanh, M. O. Nestoklon, J. Even, J. M. Jancu, M. Perrin, H. Folliot, T. Rohel, S. Tricot, A. Balocchi, D. Lagarde, X. Marie, N. Bertru, O. Durand, and A. Le Corre, Phys. Rev. B 86, 205316 (2012).

[30] C. Prohl, A. Lenz, D. Roy, J. Schuppang, G. Stracke, A. Strittmatter, U. W. Pohl, D. Bimberg, H. Eisele, and M. Dähne, Appl. Phys. Lett. 102, 123102 (2013).

[31] G. Stracke, A. Glacki, T. Nowozin, L. Bonato, S. Rodt, C. Prohl, A. Lenz, H. Eisele, A. Schliwa, A. Strittmatter, U. W. Pohl, and D. Bimberg, Appl. Phys. Lett. 101, 223110 (2012).

[32] J. Shen, Y. Song, M. L. Lee, and J. J. Cha, Nanotechnology 25, 465702 (2014).

[33] F. Fukami, K. Umeno, Y. Furukawa, N. Urakami, S. Mitsuyoshi, H. Okada, H. Yonezu, and A. Wakahara, Phys. Status Solidi C 8, 322 (2011).

[34] G. Stracke, E. M. Sala, S. Selve, T. Niermann, A. Schliwa, A. Strittmatter, and D. Bimberg, Appl. Phys. Lett. 104, 123107 (2014).

[35] C. Robert, M. O. Nestoklon, K. P. da Silva, L. Pedesseau, C. Cornet, M. I. Alonso, A. R. Goñi, P. Turban, J.-M. Jancu, J. Even, and O. Durand, Appl. Phys. Lett. 104, 011908 (2014).

[36] C. Cornet, A. Schliwa, J. Even, F. Doré, C. Celebi, A. Létoublon, E. Macé, C. Paranthoën, A. Simon, P. M. Koenraad, N. Bertru, D. Bimberg, and S. Loualiche, Phys. Rev. B 74, 035312 (2006).

[37] Q. Gong, P. Offermans, R. Nötzel, P. M. Koenraad, and J. H. Wolter, Appl. Phys. Lett. 85, 5697 (2004).

[38] A. D. Giddings, J. G. Keizer, M. Hara, G. J. Hamhuis, H. Yuasa, H. Fukuzawa, and P. M. Koenraad, Phys. Rev. B 83, 205308 (2011).

[39] P. Offermans, P. M. Koenraad, J. H. Wolter, K. Pierz, M. Roy, and P. A. Maksym, Phys. Rev. B 72, 165332 (2005).

[40] V. Mlinar, M. Bozkurt, J. M. Ulloa, M. Ediger, G. Bester, A. Badolato, P. M. Koenraad, R. J. Warburton, and A. Zunger, Phys. Rev. B 80, 165425 (2009).

[41] P. N. Keating, Phys. Rev. 145, 637 (1966).

[42] I. Vurgaftman, J. R. Meyer, and L. R. Ram-Mohan, J. Appl. Phys. 89, 5815 (2001).

[43] Z. Sui and I. P. Herman, Phys. Rev. B 48, 17938 (1993).

[44] K. Kim, P. R. C. Kent, A. Zunger, and C. B. Geller, Phys. Rev. B 66, 045208 (2002).

[45] O. L. Lazarenkova, P. von Allmen, F. Oyafuso, S. Lee, and G. Klimeck, Superlattices Microstruct. 34, 553 (2003). 
[46] S. Steiger, M. Salmani-Jelodar, D. Areshkin, A. Paul, T. Kubis, M. Povolotskyi, H.-H. Park, and G. Klimeck, Phys. Rev. B 84, 155204 (2011).

[47] M. Zieliński, Phys. Rev. B 86, 115424 (2012).

[48] J.-M. Jancu, R. Scholz, F. Beltram, and F. Bassani, Phys. Rev. B 57, 6493 (1998).

[49] D. S. Watkins, The Matrix Eigenvalue Problem: GR and Krylov Subspace Methods (Society for Industrial and Applied Mathematics, Philadelphia, PA, 2007).

[50] L.-W. Wang and A. Zunger, J. Chem. Phys. 100, 2394 (1994).

[51] A. Canning, L. W. Wang, A. Williamson, and A. Zunger, J. Comput. Phys. 160, 29 (2000).

[52] L.-W. Wang and A. Zunger, Phys. Rev. B 56, 12395 (1997).

[53] T. B. Boykin and P. Vogl, Phys. Rev. B 65, 035202 (2001).

[54] W. W. Chow, S. W. Koch, and M. Sargent, SemiconductorLaser Physics (Springer-Verlag, Berlin, Heidelberg, 1994).
[55] A. Thränhardt, C. Ell, G. Khitrova, and H. M. Gibbs, Phys. Rev. B 65, 035327 (2002).

[56] A. R. Goñi, C. Kristukat, F. Hatami, S. Dreßler, W. T. Masselink, and C. Thomsen, Phys. Rev. B 67, 075306 (2003).

[57] C. Kristukat, A. R. Goñi, K. Pötschke, D. Bimberg, and C. Thomsen, Phys. Status Solidi B 244, 53 (2007).

[58] H. K. Mao, J. Xu, and P. M. Bell, J. Geophys. Res. Solid Earth 91, 4673 (1986).

[59] A. R. Goñi and K. Syassen, in High Pressure in Semiconductors Physics I, edited by T. Suski and W. Paul, Semiconductors and Semimetal Series (Elsevier, Amsterdam, 1998), Vol. 54, Chap. 4.

[60] A. R. Goi, K. Syassen, K. Strössner, and M. Cardona, Phys. Rev. B 39, 3178 (1989).

[61] A. R. Goni, K. Strössner, K. Syassen, and M. Cardona, Phys. Rev. B 36, 1581 (1987).

[62] A. R. Goñi, L. R. Muniz, J. S. Reparaz, M. I. Alonso, M. Garriga, A. F. Lopeandia, J. Rodríguez-Viejo, J. Arbiol, and R. Rurali, Phys. Rev. B 89, 045428 (2014). 